\title{
Spin Relaxation Effects in Oil-Nanomagnetite Ferrofluids Mössbauer Spectrometry Studies
}

\author{
T. Szumiata ${ }^{a *}$, M. Gzik-Szumiata $^{a}$, K. BrzÓzkA ${ }^{a}$, B. Górka ${ }^{a}$, M. GaWroński $^{a}$,

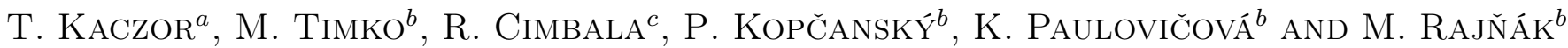 \\ ${ }^{a}$ Department of Physics, Faculty of Mechanical Engineering, University of Technology and Humanities in Radom, \\ E. Stasieckiego 54, 26-600 Radom, Poland \\ ${ }^{b}$ Institute of Experimental Physics SAS, Watsonova 47, 04001 Košice, Slovakia \\ ${ }^{c}$ Department of Electrical Power Engineering, Technical University of Košice, \\ Faculty of Electrical Engineering and Informatics, Mäsiarska 74, 04120 Košice, Slovakia
}

\begin{abstract}
Superparamagnetic effects have been studied in oil-nanomagnetite ferrofluid by means of transmission Mössbauer spectrometry. At room temperature it was necessary to slow down Brownian motions of nanoparticle by annealing which make oil more viscous. This procedure is alternative to the freezing in liquid nitrogen. The spectra were fitted with four Gaussian distributions of hyperfine field. Within the analytical model of spin relaxation based on the Liouville super-operators, the mean nanoparticles diameter as well as its standard deviation have been estimated. The reasonable agreement with transmission electron microscopy outcomes has been achieved, especially in the case of the Mössbauer spectra obtained at liquid nitrogen temperature. The additional Mössbauer spectrometry measurements at room temperature in external magnetic fields allowed to verify, if low hyperfine field Mössbauer subspectra originate indeed from the finest particles.
\end{abstract}

DOI: 10.12693/APhysPolA.134.1007

PACS/topics: 75.75.Jn, 31.30.Gs, 75.50.Dd, 75.50.Gg

\section{Introduction}

During recent decades ferrofluids focus a lot of attention due to their specific properties and potential applications in technology and medicine [1-4]. The majority of these unique properties originate from the physical phenomena in small-sized, single-domain magnetic nanoparticles suspended in liquid carrier (polar or nonpolar) and forming a magnetic colloid. These particles are responsible e.g. for very peculiar spin and dielectric relaxation processes, magnetic mesostructuring, as well as change of magnetic and electro-rheological properties under influence of the external electric field [5-10].

Main goal of this work was the observation of spin relaxation phenomena in oil-nanomagnetite-based ferrofluid by means of the Mössbauer spectrometry and the interpretation of the obtained results in terms of the simple, analytical model in order to reproduce a size distribution of particles. The motivation of undertaking such a task was the fact that in the majority of the Mössbauer papers devoted to superparamagnetic effects in nanoparticles nanoferrofluids, usually experimental data are presented without quantitative theoretical analysis. In some cases the numerical analysis models have been applied - however, without detailed description of the algorithm (e.g. [11]).

Due to Brownian motion of nanoparticles dispersed in oil the Doppler effect smeared out the Mössbauer

\footnotetext{
*corresponding author; e-mail: t.szumiata@uthrad.pl
}

spectrum for the sample of the original ferrofluid. The preliminary Mössbauer spectrometry experiment showed that neither nanoparticles densification by partial separation with magnetic field nor the applying of external magnetic field (of the magnitude up to $0.5 \mathrm{~T}$ ) during measurements were not able to slow down sufficiently the Brownian motion of nanoparticles. Thus, an annealing of ferrofluid at moderate temperature has been proposed as a way of fixing the nanoparticles for transmission Mössbauer spectrometry at room temperature (RT). These results have been compared with the spectra obtained for the original ferrofluid sample frozen in liquid nitrogen (LN).

The analytical model of spin relaxation based on superoperators formalism has been utilized for the description of superparamagnetic effects seen in the Mössbauer spectra. It has enabled to estimate the parameters of particles diameters distribution. The discrepancies between outcomes obtained from RT and LN spectra have been discussed.

\section{Samples and experimental methods}

The magnetic ferrofluid was fabricated with commercial transformer oil MOGUL ${ }^{T M}$ and magnetite nanoparticles of about 3\% mass concentration utilizing the technological procedure described in [7-9]. Examination of the prepared magnetic fluids was done using transmission electron microscopy (TEM), Tesla BS 500 microscope, normally operated at $90 \mathrm{kV}$ with 80,000 magnification by the replication technique. A drop of magnetic fluid sample diluted in water was deposited on the 400 mesh 
copper grid and air dried before the picture (Fig. 1) was taken. Final results of TEM photo was already reported in [8]. Mean diameter value of almost spherical magnetite nanoparticles was estimated as $\tilde{d}_{\mathrm{TEM}}=10 \mathrm{~nm}$. In fact, the total diameter was about $14 \mathrm{~nm}$, but there was a surfactant layer $2 \mathrm{~nm}$ thick on the nanoparticles surface. Thus in order to find a diameter of magnetite core it was necessary to subtract $2 \times 2 \mathrm{~nm}$ from the total mean diameter of nanoparticles. This estimated diameter value was confirmed by magnetic investigation with vibrating sample magnetometer (VSM). In this method (described in [5]) the magnetization curve of diluted ferrofluid in the external magnetic field is assumed to be reproduced by convolution of the Langevin function and log-normal distribution of nanoparticles diameters.

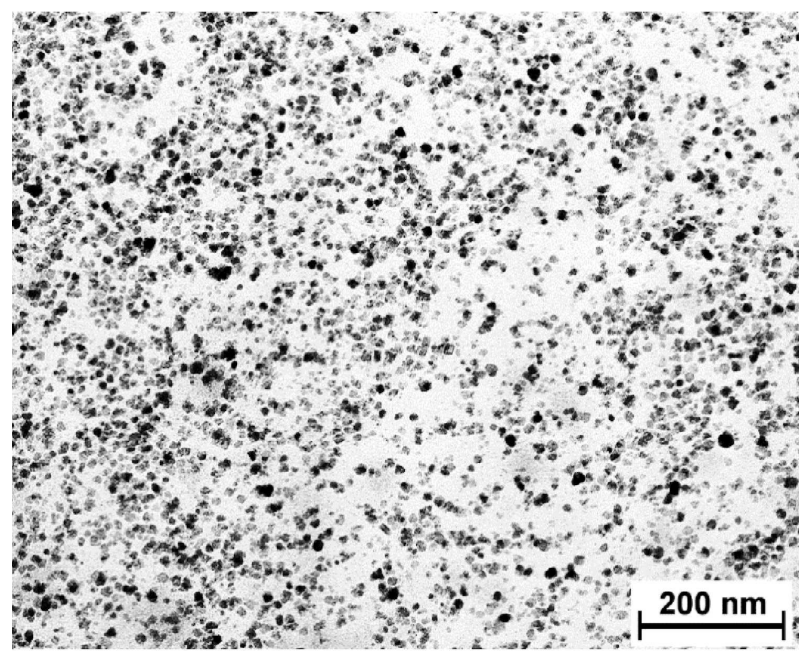

Fig. 1. TEM micrograph of air dried, oilnanomagnetite ferrofluid sample (previously diluted in water).

For the Mössbauer spectroscopy investigations the samples of the ferrofluid have undergone a partial separation process of magnetite nanoparticles from oil base using permanent magnet. This procedure was insufficient to obtain the Mössbauer spectra, because nanoparticles had still too much freedom and the spectra were smeared out via the Doppler effect. This fact gave a motivation for inventing a simple and cheap method of slowing down the nanoparticles motion. The one hour annealing at $180^{\circ} \mathrm{C}$ was applied, which transformed the oil into semiliquid, viscous substance. At the same time, the magnetite nanoparticles remained unchanged owing to properly chosen, not too high temperature of annealing.

Transmission Mössbauer spectra at RT were collected by the system arranged in vertical geometry and consisting of ${ }^{57} \mathrm{Co} / \mathrm{Rh}$ source of gamma radiation, vibrator operating in constant acceleration mode and ionization counter as a detector of $\gamma$-rays. Moreover, the Mössbauer measurements at liquid nitrogen temperature were performed by immersing the sample in a simple nitric cryostat. Additionally, the samples were also measured in an external magnetic field: (i) perpendicular to the gamma beam, with $B_{e x}=480.9 \mathrm{mT}$ and (ii) longitudinal to the gamma beam, with $B_{e x}=164.4 \mathrm{mT}$. The magnetic field was produced by a sets of two neodymium-based permanent magnets (in the case of longitudinal field, both magnets had a central holes).

The Mössbauer spectra were fitted using open source PolMöss software, which is a package based on MS Excel with Solver optimizing module realizing gradient and evolutionary (genetic) algorithms on multicore processors within parallel calculations implementation. The fitting procedure offers a convolution of the Lorentzian baseline with Gaussian distributions of hyperfine parameters resulting in the final form as the Voigt profile. The PolMöss software and fitting method was efficiently applied in processing of the Mössbauer spectra of the environmental samples like highways and industrial dusts, fly ashes from power and heat plants as well as natural soils [12-14].

\section{Theoretical model}

The superparamagnetic effects are caused by nanoparticle spin flipping, when the thermal energy is comparable to the total magnetic anisotropy energy barrier of the nanoparticle: $\Delta E(d)=K V(d)$, where $K$ is a magnetocrystalline anisotropy energy constant, $d$ - the diameter of nanoparticle, and $V(d)=1 / 6 \pi d^{3}$ is a volume of spherical nanoparticle. Within Brown theory [11, 15, 16], the relaxation time is given by the formula

$$
\tau_{R}(d)=\frac{M_{s} \sqrt{\pi}}{2 K \gamma_{0}}\left(\frac{\Delta E(d)}{k T}\right)^{-\frac{1}{2}} \mathrm{e}^{\frac{\Delta E(d)}{k T}},
$$

where $M_{s}$ is saturation magnetization, $\gamma_{0}=e / m_{e}$ gyromagnetic ratio, $k$ - the Boltzmann constant, $T$ absolute temperature. Corresponding spin flip angular frequency is equal to $\omega_{R}(d)=2 \pi / \tau_{R}(d)$.

The chaotic changes of the electronic spins in nanoparticle generate dynamic oscillations of hyperfine magnetic field acting on the ${ }^{57} \mathrm{Fe}$ nuclear magnetic momenta, and they influence the magnetically split nuclear levels. The energy of splitting caused by static hyperfine magnetic field $B_{0}$ in the transition between ground and first excited state of ${ }^{57} \mathrm{Fe}$ nucleus corresponding to the external lines in the Zeeman sextet, can be expressed be the formula: $E= \pm B_{0} \times 1.2363 \times 10^{-27} \mathrm{~J} / \mathrm{T}$. This result considers values of magnetic nuclear moments for ground and excited states. The corresponding quantum frequencies (regular and angular one, respectively) are given by $\nu=E / h$ and $\varepsilon=2 \pi \nu$ and can be interpreted as effective frequencies of the Larmor precession. The analytical model of relaxation based on super-Hamiltonian formalism (the Liouville super-operators, the Clauser-Blume model) [17-20] predicts the following relaxational modification of Zeeman splitting quantum frequencies in fluctuating hyperfine magnetic field:

$$
X(d)= \pm \sqrt{\varepsilon^{2}-\omega_{R}^{2}(d)},
$$

where $0 \leq \omega_{R}(d) \leq \varepsilon$. Owing to simple, analytical form of the final result this method is implemented in commercial Mosgraf software (by Duraj and Ruebenbauer [20]) 
for fitting of the relaxational Mössbauer spectra. The efficiency and simplicity of this approach is a consequence of the fact that super-operators formalism considers a wave functions combination both of ground and excited nuclear states as a base for the perturbation theory calculus. Unfortunately Mosgraf software, at present stage of development, offers fitting procedures only for the nanoparticles of the same size (or several discrete groups of different diameters). There is no options for continuous distribution of nanoparticle size, that is why at present work a PolMöss software (Szumiata et al. [12-14]) has been utilized due to the hyperfine parameters distribution functionality (described in previous section).

According to Eq. (2) one can easily predict a corresponding absolute value of the effective hyperfine magnetic field, modified by relaxational effects in small nanoparticles

$$
B(d)=B_{0} \sqrt{1-\left(\frac{\omega_{R}(d)}{\varepsilon}\right)^{2}} .
$$

In fact, such effective field should be defined separately for each pair of lines in the Zeeman sextet, however in further analysis we will consider only the energy and the field corresponding to outer lines. Such approach is justified in the case of highly spread spectra, which correspond to a continuous set of particles of different sizes. Since PolMöss program does not contain the specialized module for relaxational effects, the distribution of particle sizes is reconstructed basing on the hyperfine field distributions according to procedure described in the next section.

\section{Results and discussion}

Room temperature Mössbauer spectrum of the annealed sample (Fig. 2a) can be satisfactory fitted by four smeared components, representing four Gaussian distributions of hyperfine field (Fig. 2b). According to [21-23] the component $G 01$ of the highest hyperfine field can be attributed to the tetrahedral A sites with $\mathrm{Fe}^{3+}$ ions in magnetite inverse spinel structure, whereas G02 component represents $\mathrm{Fe}^{2+/ 3+}$ ions in octahedral B sites. Such obvious assignment, analogical to the bulk case, is justified only for the fraction of the biggest nanoparticles, but even then it is not entirely accurate due to the distribution of particle sizes. The manifestation of this question is seen e.g. in the ratio of $G 02$ to $G 01$ component contribution. For the stoichiometric, bulk magnetite its value is expected to be equal to 2 , for defected or partially oxidized magnetite - less than 2 , but in our case this value is greater than 2 (see Table I). Thus a component $G 02$ contains also some contribution of more fine particles. The total contribution of these two high-field components at room temperature is about $54 \%$. Next two components ( $G 03$ and $G 04$ ) of the medium and the lowest hyperfine field represent the finest fractions of the magnetite nanoparticles but in this case it is rather impossible to distinguish A-site and B-site contribution, due to significant widening of the subspectra (the ratio of standard deviation of hyperfine field distribution to its mean value is high — see Table I). The total contribution of $G 03$ and $G 04$ subspectra is around $46 \%$, but the share of $G 04$ component was the smallest (ca. 8\%). The mean value of the HMF for $G 04$ component is very small (less than $4 \mathrm{~T}$ ). Thus considering the significant relative width of the field distribution, one can admit that this component comprises also nonmagnetic contribution, usually represented by doublet. The presence of G04 component can be interpreted as the strongest impact of the superparamagnetic, relaxational effect on the finest particles. The isomer shift $I S$ (given relatively to the standard Fe foil) has been regarded as the same for each component and after fitting it takes value (Table I) close to that obtained as an average over isomer shifts for A and B components in bulk magnetite at room temperature. The fitted values of quadrupole splitting $Q S$ (common for all components) have appeared to be close to zero - as expected for bulk magnetite. Despite that, the nanoparticles were partially separated from oil with magnetic field, the magnetization direction seems to be entirely random, because the ratio $x$ of the central lines contribution in the Zeeman sextet to the contribution of inner lines is very close to 2. Apparently, after removing magnetic field and due to annealing, the system of magnetite nanoparticles entirely loses macroscopic magnetic ordering.
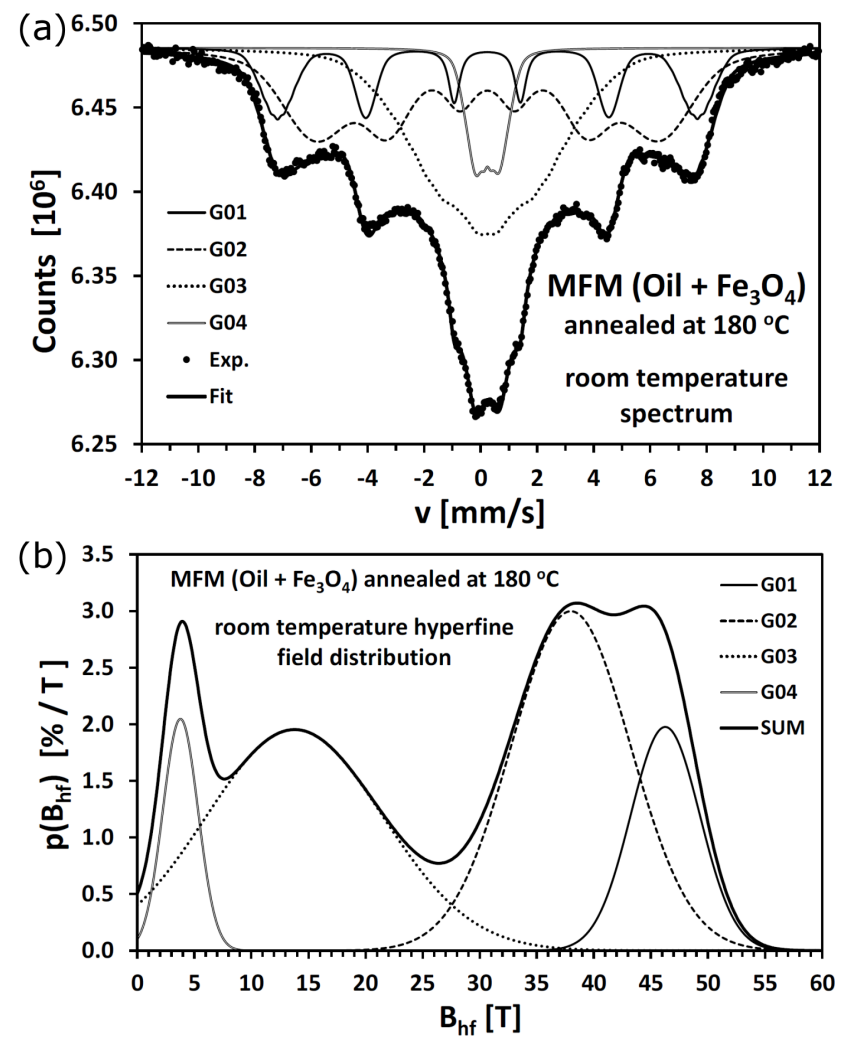

Fig. 2. Room temperature Mössbauer spectrum (a) and hyperfine field distribution (b) for the annealed sample of the oil-nanomagnetite ferrofluid. 
The hyperfine parameters of room temperature Mössbauer spectrum for the annealed sample of the oil-nanomagnetite ferrofluid.

\begin{tabular}{c|c|c|c|c|c|c|c|c|c}
\hline \hline Gauss & $p[\%]$ & $\theta\left[{ }^{\circ}\right]$ & $x$ & $I S_{0}[\mathrm{~mm} / \mathrm{s}]$ & $\sigma(I S)[\mathrm{mm} / \mathrm{s}]$ & $Q S_{0}[\mathrm{~mm} / \mathrm{s}]$ & $\left\langle B_{h f}\right\rangle[\mathrm{T}]$ & $\sigma\left(B_{h f}\right)[\mathrm{T}]$ & $\gamma / 2[\mathrm{~mm} / \mathrm{s}]$ \\
\hline$G 01$ & 15.0 & 55.6 & 2.07 & 0.348 & 0.002 & 0.006 & 46.26 & 3.03 & 0.200 \\
$G 02$ & 39.1 & 55.6 & 2.07 & 0.348 & 0.002 & 0.006 & 37.99 & 5.20 & 0.685 \\
$G 03$ & 38.0 & 55.6 & 2.07 & 0.348 & 0.002 & 0.006 & 13.79 & 7.75 & 0.522 \\
$G 04$ & 7.9 & 55.6 & 2.07 & 0.348 & 0.002 & 0.006 & 3.79 & 1.54 & 0.150
\end{tabular}

The hyperfine parameters of liquide nitrogen temperature Mössbauer spectrum for sample of

TABLE II the oil-nanomagnetite ferrofluid.

\begin{tabular}{c|c|c|c|c|c|c|c|c|c}
\hline \hline Gauss & $p[\%]$ & $\theta\left[{ }^{\circ}\right]$ & $x$ & $I S_{0}[\mathrm{~mm} / \mathrm{s}]$ & $\sigma(I S)[\mathrm{mm} / \mathrm{s}]$ & $Q S_{0}[\mathrm{~mm} / \mathrm{s}]$ & $\left\langle B_{h f}\right\rangle[\mathrm{T}]$ & $\sigma\left(B_{h f}\right)[\mathrm{T}]$ & $\gamma / 2[\mathrm{~mm} / \mathrm{s}]$ \\
\hline$G 01$ & 29.8 & 54.7 & 2.00 & 0.437 & 0.018 & -0.010 & 50.66 & 1.59 & 0.188 \\
$G 02$ & 41.5 & 54.7 & 2.00 & 0.437 & 0.018 & -0.010 & 46.76 & 3.05 & 0.366 \\
$G 03$ & 20.3 & 54.7 & 2.00 & 0.437 & 0.018 & -0.010 & 32.46 & 6.49 & 0.500 \\
$G 04$ & 8.5 & 54.7 & 2.00 & 0.437 & 0.018 & -0.010 & 8.02 & 2.67 & 0.271
\end{tabular}

In the case of the oil samples frozen in liquid nitrogen the Mössbauer spectra (Fig. 3a) and corresponding hyperfine field distributions (Fig. 3b, Table II) are significantly narrower (i.e. relative widths are considerably smaller). The contribution of low field $G 03+G 04$ components dropped from $46 \%$ at room temperature to $29 \%$ at liquid nitrogen temperature. It is a clear confirmation that these components represent the finest particles the magnetism of which is the most strongly influenced by relaxational effects at higher temperatures. The values of mean hyperfine fields at liquid nitrogen temperature are noticeably higher than at room temperature. For the case of high-field $G 01$ and $G 02$ components this change is of order dozen percent, which coincides with the temperature changes of the bulk magnetite magnetization. On the other hand, mean values corresponding to $G 03$ and $G 04$ components increased several times at nitrogen temperature. This is a clear proof that this change can not be explained only in terms of the magnetization evolution with temperature, but mostly by the significant drop of the relaxation effects influence. The isomer shift (fixed as common for all components) has higher value at low temperature due to second order Doppler effect.

The two additional spectra for annealed sample have been collected at room temperature under magnetic field. In the first case (Fig. 4a, b) external magnetic field of induction $B_{e x}=480.9 \mathrm{mT}$ has been applied perpendicular to $\gamma$-ray beam $\left(\beta=90^{\circ}\right)$. For bulk magnetite a slight diminishing of hyperfine magnetic field (HMF) would be expected, due to its opposite sense with respect to the macroscopic magnetization vector (parallel to the external magnetic field). However, for the investigated magnetite nanoparticles one observes small increase of HMF of $G 01$ component and slightly higher of $G 02$ component (Table III) - in both cases more than magnitude of external magnetic field. It can be interpreted as suppression of spin relaxation effects by applying of magnetic field. The most pronounced effect of this type is visible for $G 03$ and $G 04$ components, where HMF mean values and widths of HMF have increased several times. This is additional argument that these components correspond to the finest fraction of magnetite. Such interpretation is backed up by the remark that total contribution of G03 and G04 components diminishes in favour of the total contribution of $G 01$ and $G 02$ components. A natural consequence of applying the magnetic field perpendicular to the $\gamma$-ray beam is a significant increase of the intensity ratio $x$ of central to the inner lines in the Zeeman sextets over the 2 value characteristic for random spin arrangement. In our case it is about 3.2 , which corresponds to the mean angle $\theta \approx 71^{\circ}$ between spins (and $\mathrm{HMF}$ ) direction and beam line.
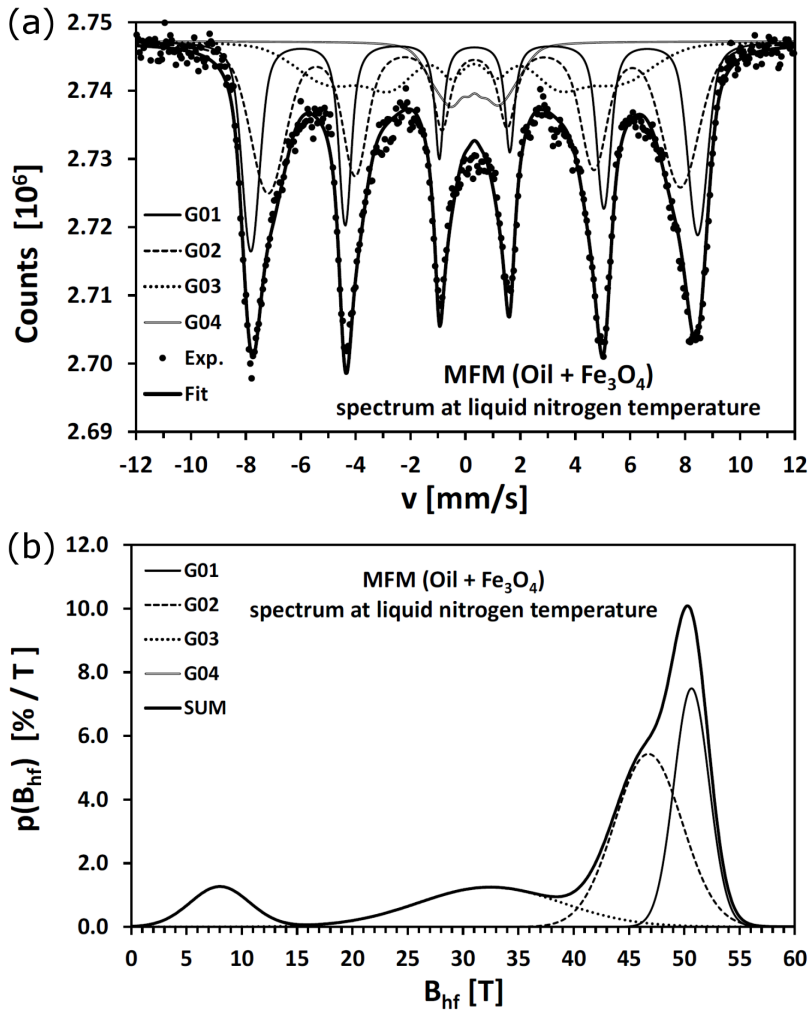

Fig. 3. Liquid nitrogen temperature Mössbauer spectrum (a) and hyperfine field distribution (b) for sample of the oil-nanomagnetite ferrofluid. 
TABLE III

The hyperfine parameters of room temperature Mössbauer spectrum for the annealed sample of the oil-nanomagnetite ferrofluid in the external magnetic field perpendicular to the $\gamma$-ray beam. The negative values of the standard deviation of the isomer shift result from the negative correlation of the isomer shift changes with corresponding changes of the hyperfine magnetic field.

\begin{tabular}{c|c|c|c|c|c|c|c|c|c}
\hline \hline Gauss & $p[\%]$ & $\theta\left[{ }^{\circ}\right]$ & $x$ & $I S_{0}[\mathrm{~mm} / \mathrm{s}]$ & $\sigma(I S)[\mathrm{mm} / \mathrm{s}]$ & $Q S_{0}[\mathrm{~mm} / \mathrm{s}]$ & $\left\langle B_{h f}\right\rangle[\mathrm{T}]$ & $\sigma\left(B_{h f}\right)[\mathrm{T}]$ & $\gamma / 2[\mathrm{~mm} / \mathrm{s}]$ \\
\hline$G 01$ & 29.4 & 70.6 & 3.21 & 0.345 & -0.023 & -0.004 & 46.98 & 2.25 & 0.211 \\
$G 02$ & 31.5 & 70.6 & 3.21 & 0.345 & -0.023 & -0.004 & 40.73 & 4.35 & 0.356 \\
$G 03$ & 34.3 & 70.6 & 3.21 & 0.345 & -0.023 & -0.004 & 29.24 & 12.45 & 1.000 \\
$G 04$ & 4.8 & 70.6 & 3.21 & 0.345 & -0.023 & -0.004 & 13.88 & 14.46 & 1.573
\end{tabular}

TABLE IV

The hyperfine parameters of room temperature Mössbauer spectrum for the annealed sample of the oil-nanomagnetite ferrofluid in the external magnetic field parallel to the $\gamma$-ray beam. The negative values of the standard deviation of the isomer shift result from the negative correlation of the isomer shift changes with corresponding changes of the hyperfine magnetic field.

\begin{tabular}{c|c|c|c|c|c|c|c|c|c}
\hline \hline Gauss & $p[\%]$ & $\left.\theta{ }^{\circ}\right]$ & $x$ & $I S_{0}[\mathrm{~mm} / \mathrm{s}]$ & $\sigma(I S)[\mathrm{mm} / \mathrm{s}]$ & $Q S_{0}[\mathrm{~mm} / \mathrm{s}]$ & $\left\langle B_{h f}\right\rangle[\mathrm{T}]$ & $\sigma\left(B_{h f}\right)[\mathrm{T}]$ & $\gamma / 2[\mathrm{~mm} / \mathrm{s}]$ \\
\hline$G 01$ & 17.3 & 36.2 & 0.85 & 0.350 & -0.016 & -0.013 & 46.88 & 1.81 & 0.235 \\
$G 02$ & 35.1 & 36.2 & 0.85 & 0.350 & -0.016 & -0.013 & 41.89 & 4.03 & 0.328 \\
$G 03$ & 20.0 & 36.2 & 0.85 & 0.350 & -0.016 & -0.013 & 28.81 & 6.54 & 0.346 \\
$G 04$ & 27.5 & 36.2 & 0.85 & 0.350 & -0.016 & -0.013 & 20.07 & 14.25 & 1.031
\end{tabular}
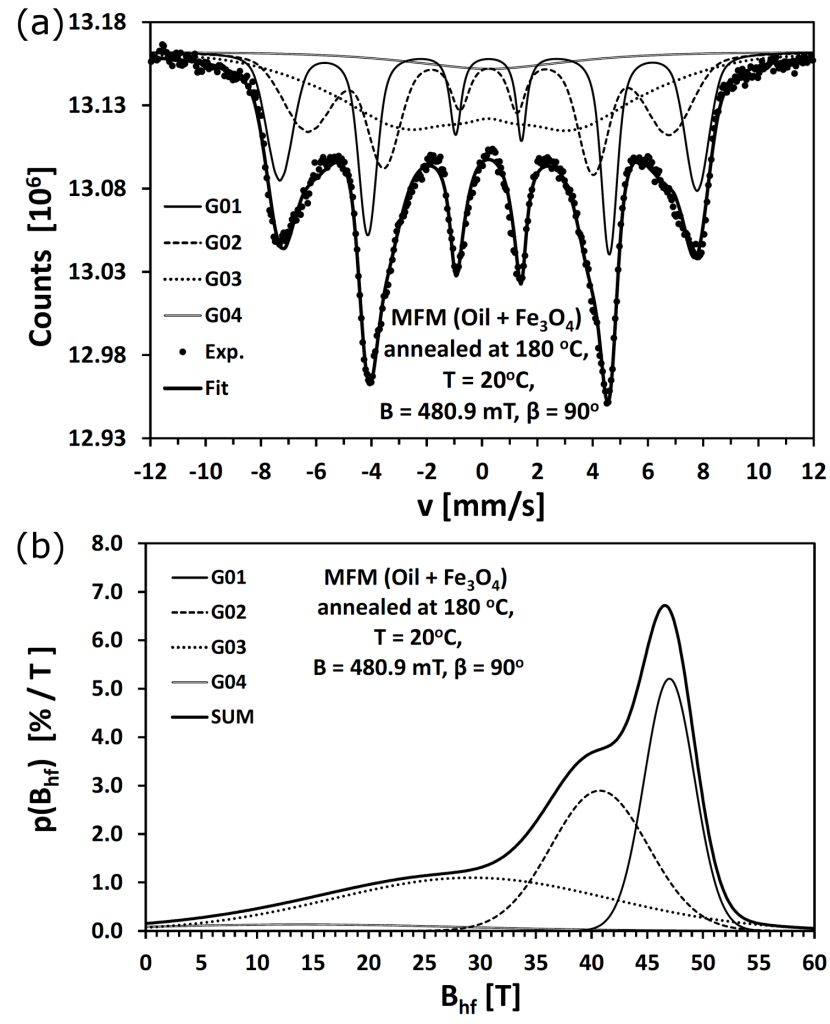

Fig. 4. Room temperature Mössbauer spectrum (a) and hyperfine field distribution (b) for the annealed sample of the oil-nanomagnetite ferrofluid in the external magnetic field perpendicular to the $\gamma$-ray beam.

Analogical remarks and conclusions are valid in the case of the spectra and HMF distributions (Fig. 5a, b) obtained when applying magnetic field of induction $B_{e x}=$ $164.4 \mathrm{mT}$ parallel to $\gamma$-ray beam $\left(\beta=0^{\circ}\right)$. The obvious difference is a reorientation of the spin mean direction $\left(\theta \approx 36^{\circ}\right)$, determined basing on the $x$ ratio value, which in this case is much lower than 2 (i.e. 0.85). Another remarkable feature is fact that despite lower value of the applied field the efficiency of nanoparticles spin ordering is even higher (Table IV). In general, for both magnetic field configurations the gained outcomes undoubtedly convinced that low-field components in the Mössbauer spectra really correspond to the finest fraction of magnetite nanoparticles - i.e. not to any other phase. However, the reduction of mean hyperfine magnetic field could be caused in part by structural and magnetic disorder at the surface of nanoparticles - not only by pure spin relaxational effects.

The final goal of this work was to estimate the mean values nanoparticles sizes (or even finding their distribution) basing on the Mössbauer spectra and spin relaxation model described in Sect. 3. According to the formulae (1) and (3), the mean diameters of nanoparticles $d(\langle B\rangle)$ have been calculated, which correspond to mean hyperfine value $\langle B\rangle$ of each component of the Mössbauer spectra. The results both for the annealed sample measured at room temperature and for the frozen sample in liquid nitrogen have been collected in Table V. For simplicity a value of magnetocrystalline anisotropy constant just for bulk magnetite at room temperature has been considered $\left(K=1.1 \times 10^{4} \mathrm{~J} / \mathrm{m}^{3}\right)$ [24] for all calculations. The saturation magnetization of bulk magnetite at room temperature has been taken as $M_{s}=30560 \mathrm{~A} / \mathrm{m}$ (estimated from data available in [25]) and rescaled for liquid nitrogen temperature basing on thermomagnetic curves for spontaneous magnetization magnetite. The room temperature $B_{0}$ values of non-perturbed HMF for $\mathrm{A}$ and B sites ( $G 01$ and $G 02$ component, respectively) in bulk magnetite have been put into formula (3) from standard catalogue of hyperfine parameters of minerals [26]. 

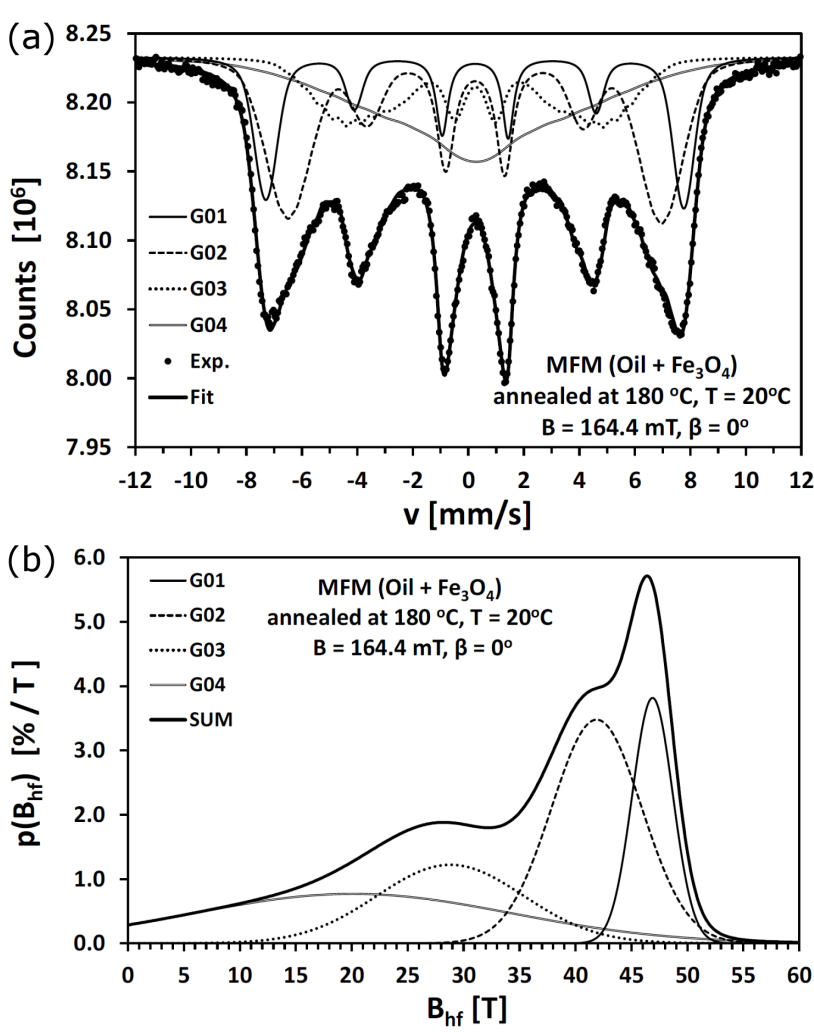

Fig. 5. As in Fig. 4, but parallel to the $\gamma$-ray beam.

For the nitrogen temperature case it has been renormalized according to thermomagnetic curves. For $G 03$ and $G 04$ more smeared components (each represents a mixture of subspectra of A and B sites for the finest nanoparticles fractions) it has been assumed that common (averaged) value of non-perturbed HMF is equal to $B_{0}=\left(B_{0 A}+2 B_{0 B}\right) / 3$, when considering that Fe ions are distributed in two sites identically like in stoichiometric, bulk magnetite.

The results of calculations presented in Table $\mathrm{V}$ demonstrate that averaged diameter of nanoparticles estimated for all fractions ranges from $16.3 \mathrm{~nm}$ to $15.3 \mathrm{~nm}$, when considering room temperature spectra for the annealed samples. This result does not coincide well with TEM and magnetic outcomes $(\tilde{d}=10 \mathrm{~nm})$. The possible reason of this discrepancy is size dependence of magnetocrystalline anisotropy constant $K$. There are some attempts of determining experimentally $K$ values for different size of magnetite nanoparticles, however in [27] the values reported for two different diameters (just above and below $10 \mathrm{~nm}$ ) where order of magnitude higher than the value for bulk. Such result seems to be not physical, because any known surface effects rather could not lead to such increase of the effective anisotropy energy. Moreover, when considering these two reported $K$ values for magnetite nanoparticles and size dependence of $K$ as proportional to the reciprocal of diameter, one obtain bulk $K$ estimation value of sign opposite to real, experimental value. Finally, instead of model improvement, in this case one obtains from Eq. (1) and (3) the diameters val- ues twice smaller than those known from TEM. Thus for further analysis we decided to leave $K$ value corresponding to room temperature and bulk material. It is worth mention that the relatively narrow range of nanoparticle diameters contrasts with very wide spread of HMF distributions. However, it is a simple consequence of the fact that dependence of the relaxation time $\tau_{R}$ on the nanoparticle diameter is extremely fast (Eq. (1)).

The analysis of the Mössbauer spectra obtained at liquid nitrogen temperature allowed to obtain $d(\langle B\rangle)$ values ranging from $10.1 \mathrm{~nm}$ to $9.4 \mathrm{~nm}$ (Table V), which is in very good agreement with TEM outcomes. Presumably, the temperature and size dependences of the effective $K$ constant mutually compensate, thus the bulk value for room temperature becomes the most suitable in this case. Anyway, if one intends to utilize spin relaxation effects seen in the Mössbauer spectra as a systematic tool for determining the nanoparticle size distributions in oil-based ferrofluids (instead of TEM, demanding complex samples preparation), it is obligatory to make a prior "calibration" of $K$ value, basing on TEM outcomes for several "standard" samples at given temperature and for given size range.

In order to increase the precision of the determining the size distributions of nanoparticles (including mean values and widths) one should consider asymmetry of the $d(\langle B\rangle)$ dependence originating from the specific form of Eqs. (1) and (3). This asymmetry manifests in the strong difference between $d\left(\langle B\rangle+\sigma_{B} / 2\right)$ and $d\left(\langle B\rangle-\sigma_{B} / 2\right)$ quantities (see Table V), where $\sigma_{B}$ is a standard deviation of HMF distribution (as a measure of its width) for each component in the Mössbauer spectrum. In this approach it is possible to correct the calculations of average values of nanoparticles in each fraction assuming that $d_{\mathrm{avr}}=\left[d\left(\langle B\rangle+\sigma_{B} / 2\right)+d\left(\langle B\rangle-\sigma_{B} / 2\right)\right] / 2$. As visible in Table $\mathrm{V}$, the largest differences between corrected $d_{\text {avr }}$ diameters and initial $d(\langle B\rangle)$ values are in the case of the coarse fraction of nanoparticles. In order to estimate the mean value over whole ensemble for nanoparticles one can utilize the following formula:

$\tilde{d}=\sum_{i=G 01}^{G 04} \frac{p_{i}}{2}\left[d\left(\left\langle B_{i}\right\rangle-\frac{\sigma_{B_{i}}}{2}\right)+d\left(\left\langle B_{i}\right\rangle+\frac{\sigma_{B_{i}}}{2}\right)\right]$,

in which summation runs over all components (form $G 01$ to $G 04$ ), and $p_{i}$ denotes the contribution of given component (Table V). Analogically, one can also define the width ("standard deviation") of the diameters distribution of all nanoparticles:

$$
\begin{aligned}
\sigma_{d} & =\left\{\sum _ { i = G 0 1 } ^ { G 0 4 } \frac { p _ { i } } { 2 } \left[\left(d\left(\left\langle B_{i}\right\rangle-\frac{\sigma_{B_{i}}}{2}\right)-\tilde{d}\right)^{2}\right.\right. \\
& \left.\left.+\left(d\left(\left\langle B_{i}\right\rangle+\frac{\sigma_{B_{i}}}{2}\right)-\tilde{d}\right)^{2}\right]\right\}^{\frac{1}{2}} .
\end{aligned}
$$

The values of $\tilde{d}$ and $\sigma_{d}$ have been calculated both from the room temperature and liquid nitrogen spectra and collected in Table V. 
TABLE V

The parameters of hyperfine field distributions and sizes distributions of nanoparticles determined from the analysis of superparamagnetic effects seen in the Mössbauer spectra for the annealed oil-nanomagnetite ferrofluid at room temperature (RT) and for the sample frozen in liquid nitrogen (LN).

\begin{tabular}{|c|c|c|c|c|c|c|c|c|c|c|}
\hline$T$ & Gauss & $\begin{array}{c}\langle B\rangle-\sigma / 2 \\
{[\mathrm{~T}]}\end{array}$ & $\begin{array}{c}\langle B\rangle \\
{[\mathrm{T}]}\end{array}$ & $\begin{array}{c}\langle B\rangle+\sigma / 2 \\
{[\mathrm{~T}]}\end{array}$ & $\begin{array}{c}d(\langle B\rangle-\sigma / 2) \\
{[\mathrm{nm}]}\end{array}$ & $\begin{array}{c}d(\langle B\rangle) \\
{[\mathrm{nm}]}\end{array}$ & $\begin{array}{c}d(\langle B\rangle+\sigma / 2) \\
{[\mathrm{nm}]}\end{array}$ & $\begin{array}{l}d_{\mathrm{avr}} \\
{[\mathrm{nm}]}\end{array}$ & $p_{i}$ & \\
\hline \multirow{4}{*}{$\mathrm{RT}$} & $G 01$ & 44.75 & 46.26 & 47.78 & 16.163 & 16.373 & 16.748 & 16.456 & 15.0 & $\tilde{d}$ \\
\hline & $G 02$ & 35.39 & 37.99 & 40.60 & 15.770 & 15.900 & 16.093 & 15.932 & 39.1 & $15.719[\mathrm{~nm}]$ \\
\hline & $G 03$ & 9.91 & 13.79 & 17.66 & 15.275 & 15.301 & 15.336 & 15.306 & 37.9 & $\sigma_{d}$ \\
\hline & $G 04$ & 3.02 & 3.79 & 4.56 & 15.251 & 15.253 & 15.254 & 15.253 & 7.9 & $0.452[\mathrm{~nm}]$ \\
\hline \multirow{4}{*}{$\mathrm{LN}$} & $G 01$ & 49.87 & 50.66 & 51.46 & 10.001 & 10.051 & 10.109 & 10.055 & 29.8 & $\tilde{d}$ \\
\hline & $G 02$ & 45.24 & 46.76 & 48.28 & 9.965 & 10.048 & 10.159 & 10.062 & 41.4 & $9.916[\mathrm{~nm}]$ \\
\hline & $G 03$ & 29.21 & 32.45 & 35.70 & 9.567 & 9.608 & 9.657 & 9.612 & 20.3 & $\sigma_{d}$ \\
\hline & $G 04$ & 6.68 & 8.02 & 9.35 & 9.435 & 9.439 & 9.442 & 9.439 & 8.5 & $0.241[\mathrm{~nm}]$ \\
\hline
\end{tabular}

In many cases it is usually assumed that size dispersion of the nanoparticles is described by log-normal distribution

$$
P(d)=\frac{1}{\sqrt{2 \pi} d s} \exp \left(\frac{-\ln ^{2}\left(\frac{d}{d_{0}}\right)}{2 s^{2}}\right)
$$

with two parameters $d_{0}$ and $s$ related to the mean value $\tilde{d}$ and standard deviation $\sigma_{d}$ by the following formulae:

$$
\begin{aligned}
& d_{0}=\frac{\tilde{d}}{\sqrt{1+\left(\frac{\sigma_{d}}{\tilde{d}}\right)^{2}}}, \\
& s=\sqrt{\ln \left[1+\left(\frac{\sigma_{d}}{\tilde{d}}\right)^{2}\right]} .
\end{aligned}
$$

In the case of small size dispersion (narrow distribution) the parameter $d_{0}$ approaches the mean value. The $s$ parameter is a dimensionless quantity and approaches zero when the ratio of standard deviation to the mean value is small. Moreover, mean value $\tilde{d}$ and $s$ parameter are related via the formula $d_{m}=\tilde{d} \mathrm{e}^{-s^{2}}$ with $d_{m}$ diameter corresponding to the maximum value of probability density of log-normal distribution.

The log-normal distributions obtained by the described procedure are presented in Fig. 6a and b, for the case of RT and LN measurements, respectively. As it has been already mentioned, the good agreement of the estimated mean values of nanoparticles from individual fractions with that obtained from TEM has been observed in the case of samples frozen in liquid nitrogen, whereas for annealed samples and measured at room temperature we stated a significant discrepancy. This tendency is also confirmed when looking at mean values $\tilde{d}$ estimated for whole nanoparticles ensemble (Table V). The already suggested reason of this facts is probably not well known dependence of magnetocrystalline anisotropy energy constant of magnetite nanoparticles on size and temperature. Moreover, as seen in Fig. $1 \mathrm{a}$ and $\mathrm{b}$ as well as in Table $\mathrm{V}$ the estimated width (standard deviation) of size distribution is twice greater in the case of room temperature measurements. The possible explanation of this feature is a specifics of spin relaxation effects which cause not only the drop of the effective HMF but also widen HMF distribution, however considering of this question exceeds the frame of the analysis intended in present work. Another unresolved problem influencing the quality of presented method is a strict determination of the number of components in the Mössbauer spectra below the Verwey transition in magnetite ( $c a .125 \mathrm{~K}$ - for bulk), as well as principle problems in determination of this transition temperature in fine nanoparticles [21, 22].
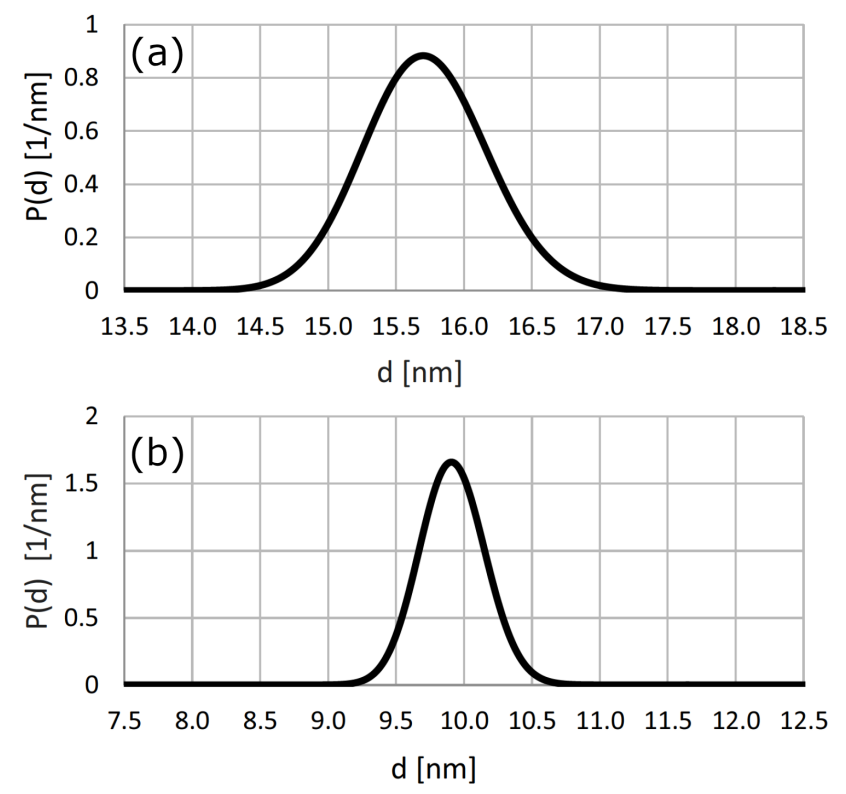

Fig. 6. Log-normal distributions of nanoparticles sizes in oil-nanomagnetite ferrofluid determined from the analysis of superparamagnetic effects seen in the Mössbauer spectra collected at (a) room temperature (after annealing), (b) liquid nitrogen temperature.

\section{Conclusions}

The performed Mössbauer spectroscopy investigations of oil-based ferrofluid with magnetite nanoparticles have demonstrated that partial magnetic separation (leading to the densification of particles) and measurement in 
magnetic field is not sufficient to slow down the Brownian motion of magnetite nanoparticles (of averaged size ca. $10 \mathrm{~nm}$ ) dispersed in oil medium. In order to obtain the Mössbauer spectra the specimens must be frozen in liquid nitrogen during measurements or be appropriately annealed before measurements. Annealing at $180^{\circ} \mathrm{C}$ appeared to be an effective and cheap way for making the oil viscous enough to stop the Brownian motion and to get the Mössbauer spectra at room temperature. The spin relaxation analytical model based on the Liouville super-operators has allowed to analyze the superparamagnetism of magnetite nanoparticles in terms of reduction of the effective hyperfine magnetic fields. The spin relaxation spectra were effectively fitted with PolMöss software using several Gaussian distributions of hyperfine fields. The simple procedure has been proposed, which enables to reproduce the distribution of nanoparticles sizes as well as to determine their mean diameter and standard deviation. Such procedure could be recommended as an alternative to the standard TEM, which demands special preparation and a lot of care in the case of oil-based ferrofluids. However, the better estimation quality of mean diameter of nanoparticles has been achieved in the case of the Mössbauer spectroscopy outcomes got at liquid nitrogen temperature when magnetic particles are not only totally fixed but also spin relaxation effects are significantly reduced. The probable reason for this difference between estimations at RT and LN could be a specific dependence of the effective magnetocrystalline energy constant both on size of the particles and on the temperature. Thus, it is reasonable to adjust ("calibrate") this parameter experimentally, by comparison with one testing result of mean particles size obtained with TEM. The additional Mössbauer measurements in the external magnetic fields confirmed, that low hyperfine magnetic field components in the spectra can be assigned to the finest fraction of the nanoparticles. The quantitative analysis of an influence of the external magnetic field on the spin relaxation processes, as well as influence of superparamagnetic effects on widening the Mössbauer spectra demand the extension of the theoretical model and can be the subjects of future investigations.

\section{Acknowledgments}

This work was supported by the University of Technology and Humanities in Radom under statutory grant $3179 / 25 / \mathrm{P}$. The authors of the article submit posthumous expressions of the gratitude to Prof. Krzysztof Ruebenbauer, who thanks to many discussions and advice, had a great influence on the substantive form of this article.

\section{References}

[1] J. Popplewell, Phys. Technol. 15, 150 (1984).

[2] C. Scherer, A.M. Figueiredo Neto, Brazil. J. Phys. 35, 718 (2005).
[3] T.-H. Tsai, L.-S. Kuo, P.-H. Chen, D.-S Lee, Ch.-T. Yang, Sensors 10, 8161 (2010).

[4] M.P. Marszałł, Pharm. Res. 28, 480 (2011).

[5] Z. Rozynek, A. Józefczak, K.D. Knudsen, A. Skumiel, T. Hornowski, J.O. Fossum, M. Timko, P. Kopčanský, M. Koneracká, Eur. Phys. J. E 34, 28 (2011).

[6] M. Rajnak, J. Kurimsky, B. Dolnik, K. Marton, L. Tomco, A. Taculescu, L. Vekas, J. Kovac, I. Vavra, J. Tothova, P. Kopcansky, M. Timko, J. Appl. Phys. 114, 034313 (2013).

[7] M. Rajnák, J. Kurimský, B. Dolník, R. Cimbala, K. Paulovicová, P. Kopcanský, M. Timko, Acta Phys. Pol. A 131, 943 (2017).

[8] M. Rajnák, B. Dolník, J. Kovác, K. Paulovicová, Z. Mitróová, J. Kurimský, R. Cimbala, P. Kopcanský, M. Timko, Acta Phys. Pol. A 131, 887 (2017).

[9] K. Paulovicová, J. Tóthová, M. Rajnák, M. Timko, P. Kopcanský, V. Lisý, Acta Phys. Pol. A 131, 1141 (2017).

[10] M. Kosterec, J. Kurimský, R. Cimbala, M. Špes, R. Farkaš, B. Dolník, M. Gamcová, M. Rajnák, M. Timko, P. Kopcanský, K. Paulovicová, B. Vargová, Acta Phys. Pol. A 133, 570 (2018).

[11] S. Mørup, H. Topsøe, J. Lipka, J. Phys. (France) 37, C6-287 (1976).

[12] T. Szumiata, M. Rachwał, T. Magiera, K. Brzózka, M. Gzik-Szumiata, M. Gawroński, B. Górka, J. Kyzioł-Komosińska, Nukleonika 62, 187 (2017).

[13] T. Szumiata, M. Gawroński, B. Górka, K. Brzózka, R. Świetlik, M. Trojanowska, M. Strzelecka, Nukleonika 58, 107 (2013).

[14] T. Szumiata, M. Gzik-Szumiata, K. Brzózka, B. Górka, M. Gawroński, R. Świetlik, M. Trojanowska, Nukleonika 60, 151 (2015).

[15] W.F. Jr. Brown, Phys. Rev. 130, 1677 (1963).

[16] A. Aharoni, Phys. Rev. 135, A447 (1964).

[17] G.J. Long, Mössbauer Spectroscopy Applied to Inorganic Chemistry, Springer Science \& Business Media, Google Books, 2013.

[18] M. Blume, Phys. Rev. 174, 351 (1968).

[19] M.J. Clauser, M. Blume, Phys. Rev. B 3, 583 (1971).

[20] Ł. Duraj, K. Ruebenbauer, Acta Phys. Pol. A 119, 75 (2011)

[21] F.J. Berry, S. Skinner, M.F. Thomas, J. Phys. Condens. Matter 10, 215 (1998).

[22] Hien-Yoong Hah, M.Sc. Thesis, University of Tennessee, Knoxville 2016.

[23] R.R. Gabbasov, V.M. Cherepanov, M.A. Chuev, M.A. Polikarpov, V.Y. Panchenko, Hyperfine Interact. 226, 383 (2014).

[24] G. Bate, Ferromagnetic Materials, North-Holland, Amsterdam 1980.

[25] A.H. Morrish, The Physical Principles of Magnetism, Wiley, New York 1965.

[26] J.G. Stevens, A.M. Khasanov, J.W.B. Miller, H. Pollak, Z. Li, Mössbauer Mineral Handbook, Mössbauer Effect Data Center, Asheville 2005.

[27] D. Caruntu, G. Caruntu, Ch.J. O'Connor, J. Phys. D Appl. Phys. 40, 5801 (2007). 